\title{
HPV Types 16/18 E6/E7-Adenoviral Transduced Autologous Lymphocytes/alpha-Galactosylceramide Vaccine BVAC-C
}

National Cancer Institute

\section{Source}

National Cancer Institute. HPV Types 16/18 E6/E7-Adenoviral Transduced Autologous

Lymphocytes/alpha-Galactosylceramide Vaccine BVAC-C. NCI Thesaurus. Code C131495.

An immunotherapeutic vaccine composed of the immunoadjuvant alpha-

galactosylceramide (a-GC) and autologous antigen presenting cells (APCs), specifically Blymphocytes and monocytes transfected with an adenoviral vector that expresses the tumor-associated antigens (TAAs) E6 and E7 derived from human papillomavirus (HPV) types 16 and 18 (HPV-16/18 E6/E7), with potential immunostimulating and antineoplastic activities. Upon administration of BVAC-C, the APCs stimulate the immune system to mount a TAA-specific cytotoxic T-lymphocyte (CTL) response, as well as natural killer (NK) cell, NK T-cell (NKT), helper T-cell and antibody-mediated immune responses, against the tumor cells. This directly or indirectly kills the TAA-expressing tumor cells. HPV-16/18 E6/E7 are overexpressed on certain tumor cell types and play key roles in tumor cell proliferation. a-GC, an NKT cell ligand, is used to specifically stimulate NKT cells and to further stimulate an anti-tumor immune response. 